\title{
Sialolito em ducto da glândula submandibular: Relato de caso
}

\author{
Sialolith in submandibular gland duct: Case report \\ Sialolito en conducto de la glándula submandibular: Reporte de caso
}

\author{
Wanderson Thalles de Souza Braga \\ ORCID: https://orcid.org/0000-0003-3617-3950 \\ Centro Universitário Tiradentes, Brasil \\ E-mail: wanderson.thalles@souunit.com.br \\ Edson Philippe Bezerra Balbino \\ ORCID: https://orcid.org/0000-0003-3035-9199 \\ Universidade Federal de Alagoas, Brasil \\ E-mail: edsonphilippebb@hotmail.com \\ Catarina Rodrigues Rosa de Oliveira \\ ORCID: https://orcid.org/0000-0001-9178-8902 \\ Centro Universitário Cesmac, Brasil \\ E-mail: catarinarosaodonto@ hotmail.com \\ José Itamar de Omena Mateus Rocha \\ ORCID: https://orcid.org/0000-0003-2397-1742 \\ Centro Universitário Tiradentes, Brasil \\ E-mail: itamar-mateus@ hotmail.com \\ Jaqueline Farias Barbosa Costa \\ ORCID: https://orcid.org/0000-0003-2901-9832 \\ Centro Universitário Cesmac, Brasil \\ E-mail: jaquefbc@hotmail.com \\ Vanessa de Carla Batista dos Santos \\ ORCID: https://orcid.org/0000-0002-3267-3969 \\ Centro Universitário Cesmac, Brasil \\ E-mail: vanessadecarlla@yahoo.com.br \\ Sonia Maria Soares Ferreira \\ ORCID: https://orcid.org/0000-0002-4825-171X \\ Centro Universitário Cesmac, Brasil \\ E-mail: sonia.ferreira@cesmac.edu.br \\ Camila Maria Beder Ribeiro Girish Panjwani \\ ORCID: https://orcid.org/0000-0003-0203-3079 \\ Centro Universitário Cesmac, Brasil \\ E-mail: camilabeder@hotmail.com \\ Áurea Valéria de Melo Franco \\ ORCID: https://orcid.org/0000-0002-5966-8617 \\ Centro Universitário Cesmac, Brasil \\ E-mail: aurea.franco@cesmac.edu.br
}

\begin{abstract}
Resumo
A sialolitíase é uma doença não neoplásica mais comum que afeta as glândulas salivares, atribuída a obstrução de glândulas salivares ou seu ducto, pela formação de um ou mais cálculos. É uma doença que ocorre mais em glândulas salivares maiores, sendo a submandibular a mais acometida. A etiologia pode estar associada ao $\mathrm{pH}$ básico e a anatomia dos ductos bastante tortuosos e ascendentes. Descrever o caso clínico de um sialolito de extensa dimensão em glândula submandibular e o diagnóstico através de exames clínicos com auxílio de exames de imagem. Paciente gênero feminino, compareceu ao Serviço de diagnóstico bucal da extensão de estomatologia - SERPROBEM do Centro Universitário Cesmac para avaliação, queixando-se de lesão no assoalho bucal esquerdo. Foram solicitados exames complementares de imagem: ultrassonografia, telerradiografia lateral e radiografia convencional (panorâmica e oclusal), tendo a presença de massa mineralizada em região submandibular. Foi realizada excisão cirúrgica, com preservação da glândula submandibular. Com a associação de exames clínicos e de imagem, pode-se facilmente identificar casos de sialolitíase. Palavras-chave: Cálculos das glândulas salivares; Doenças da glândula submandibular; Diagnóstico por imagem.
\end{abstract}

\section{Abstract}

Sialolithiasis is a more common non-neoplastic disease that affects the salivary glands, attributed to the obstruction of salivary glands or their duct, by the formation of one or more stones. It is a disease that occurs more in larger salivary glands, with the submandibular one being the most affected. The etiology may be associated with the basic $\mathrm{pH}$ and the anatomy of the tortuous and ascending ducts. To describe the clinical case of an extensive sialolith in the submandibular gland and the diagnosis through clinical examinations with the aid of imaging tests. A female patient, attended the Oral 
Diagnosis Service of Stomatology Extension - SERPROBEM at Centro Universitário Cesmac for evaluation, complaining of a lesion in the left oral floor. Complementary imaging tests were requested: ultrasound, lateral teleradiography and conventional radiography (panoramic and occlusal), with the presence of a mineralized mass in the submandibular region. Surgical excision was performed, with preservation of the submandibular gland. With the association of clinical and imaging tests, it is easy to identify cases of sialolithiasis.

Keywords: Salivary gland calculi; Submandibular gland diseases; Diagnostic imaging.

\section{Resumen}

La sialolitiasis es una enfermedad no neoplásica más común que afecta a las glándulas salivales, atribuida a la obstrucción de las glándulas salivales o de su conducto, por la formación de uno o más cálculos. Es una enfermedad que se presenta más en glándulas salivales de mayor tamaño, siendo la submandibular la más afectada. La etiología puede estar asociada con el pH básico y la anatomía de los conductos tortuosos y ascendentes. Describir el caso clínico de un sialolito extenso en la glándula submandibular y el diagnóstico a través de exámenes clínicos con la ayuda de pruebas de imagen. Caso clínico: Paciente de sexo femenino, que acudió al Servicio de Diagnóstico Oral de Extensión de Estomatología - SERPROBEM del Centro Universitário Cesmac para valoración, por lesión en suelo oral izquierdo. Se solicitaron pruebas de imagen complementarias: ecografía, telerradiografía lateral y radiografía convencional (panorámica y oclusal), con presencia de masa mineralizada en región submandibular. Se realizó exéresis quirúrgica, con preservación de la glándula submandibular. Con la asociación de pruebas clínicas y de imagen, es fácil identificar casos de sialolitiasis.

Palabras clave: Cálculos de las glándulas salivales; Enfermedades de la glándula submandibular; Diagnóstico por imagen.

\section{Introdução}

As glândulas salivares são exócrinas, produtoras de saliva, onde mostra a sua importância na manutenção da umidade bucal, digestão de alimentos e prevenção da doença cárie. A sialolitíase é uma doença não neoplásica (Purcell et al, 2017) mais comum que afeta as glândulas salivares (Folchini \& Stolz, 2016; Purcell et al, 2017) que compromete 12 em cada 1000 indivíduos (Aiyekomgbon, Babatundle \& Salam, 2018). Ela pode ser atribuída a obstrução da glândula ou ducto excretor pela formação de um ou mais cálculos calcificados, afetando mais as glândulas salivares maiores do que as menores (Jacome \& Abdo, 2010; Abe, Kurita, Hayashi \& Minagawa,2019).

A maioria dos casos de sialolítiase provém das glândulas submandibulares (Neville, Damm, Allen \& Bouquot, 2009; Goes, Lima, Carvalho, Queiroz \& Camargo, 2013), sendo (92\%) de ocorrência, seguido pela parótida (6\%), sublingual (2\%) e glândulas salivares menores (2\%) (Abe, Kurita, Hayashi \& Minagawa,2019). A glândula submandibular tem composição salivar mista, exibindo ácinos seromucosos com secreção mais densa, rica em fósforo e pH alcalino (Kondo et al, 2018), e isso em conjunto com a anatomia sinuosa e em ascendência do ducto (Folchini \& Stolz, 2014), facilita a formação dos cálculos salivares (Kondo et al, 2018).

A sialolítiase raramente afeta crianças (Cobos, Muñoz \& Diaz, 2009), e sua maior ocorrência é em indivíduos com idade média da quinta década de vida, sexo masculino, sem predileção de raça e é caracterizado por uma volumização na região da glândula afetada (Neville, Damm \& Allen, 2009). Clinicamente apresenta uma tumefação associada com sintomatologia dolorosa, com episódios de volumização e dificuldade de excreção salivar, levando a inflamação e até mesmo a uma infecção desta glândula (Araujo \& Araújo, 1984).

A associação de exame clínico a exames complementares de imagem são os mais utilizados para diagnóstico (Folchini \& Stolz, 2014). Estes exames de imagem incluem radiografia convencional, sialografia por subtração digital, ultrassonografia, tomografia computadorizada e sialografia por ressonância magnética (Purcell et al, 2017). A radiografia panorâmica, e principalmente a oclusal, mostram as melhores imagens para o seu reconhecimento (Oteri, Procopio and Cicissiù, 2011).

Para diagnóstico definitivo do sialolito, os cortes histológicos revelam fragmentos de material eosinofílico amorfo exibindo linhas de calcificações concêntricas (Araújo, Júnior, Landin \& Fernandes, 2011).

A escolha de tratamento depende das características do cálculo (Folchini \& Stolz, 2014; Silva, Carneiro, Arantes, Louro \& Rezende, 2020). sialolito de menor dimensão quando localizados no ducto da glândula, podem ser extraído por meio de 
ordenha bimanualmente, e os maiores, por meio de acesso cirúrgico intra ou extraoral a depender da sua dimensão e localização (Folchini \& Stolz, 2014). A excisão do cálculo no interior do ducto da glândula submandibular, há necessidade de uma avaliação cuidadosa da imagem para remoção, onde surgem sem a sintomatologia, apresentando a funcionalidade normal da glândula salivar (McGurk, Makdissi \& Brown, 2004), os sialolitos submandibulares extensos, devem ser investigados com uma abordagem apropriada para evitar possíveis complicações no pós-operatório (Alkurt \& Peker, 2009).

Este trabalho tem como objetivo relatar um caso clínico de cálculo salivar no interior do ducto da glândula submandibular, que foi diagnosticado por meio de exame clínico associado a exames complementares de imagem e confirmado com o anatomopatológico.

\section{Metodologia}

O presente estudo se propõe a apresentar uma descrição do diagnóstico clínico do sialolito juntamente com os exames complementares de imagem e anatomopatológica por meio de relato de caso clínico (estudo de caso) com objetivos descritivos e uma abordagem qualitativa (Pereira et al., 2018). Assim, de acordo com o conhecimento da prática estomatológica e com base na literatura científica sobre o tema abordado, e considerando a uma sequência de condução de diagnóstico do caso apresentado, propôs-se relatar um caso clínico de cálculo salivar no interior do ducto da glândula submandibular, sendo diagnosticado por meio de exame clínico associado a exames complementares de imagem e histopatológico. O estudo teve declínio do TCLE e aprovado com o número de parecer 4.609.648.

\section{Relato de Caso}

Paciente do sexo feminino, melanoderma, 43 anos, compareceu ao Serviço de diagnóstico bucal da extensão de estomatologia - SERPROBEM do Centro Universitário Cesmac para avaliação, queixando-se de ter um "problema na língua". Extraoralmente, nada digno de nota. No exame intra-oral foi observado lesão localizada em assoalho bucal do lado esquerdo na altura de incisivo central até o segundo pré-molar, coloração normal, consistência firme, base séssil, medindo 3,0 x 1,0 x 1,0 cm com sintomatologia dolorosa (Figura 1). A hipótese clínica foi de sialoadenite/sialolitíase. Foram solicitados exames complementares de imagem; raio-x convencional: panorâmica (Figura 2) e oclusal (Figura 3), telerradiografia lateral (Figura 4) e ultrassonografia (Figura 5). Realizou excisão cirúrgica da lesão e respectivamente o tratamento com preservação da glândula envolvida por meio de biópsia do tipo excisional. Os cortes histopatológicos revelaram fragmentos de material eosinofílico amorfo exibindo linhas de calcificações concêntricas, confirmando o diagnóstico de sialólitiase (Figura 6). 
Research, Society and Development, v. 10, n. 6, e13410615607, 2021

(CC BY 4.0) | ISSN 2525-3409 | DOI: http://dx.doi.org/10.33448/rsd-v10i6.15607

Figura 1. Exame intra- oral, lesão localizada em assoalho bucal do lado esquerdo.

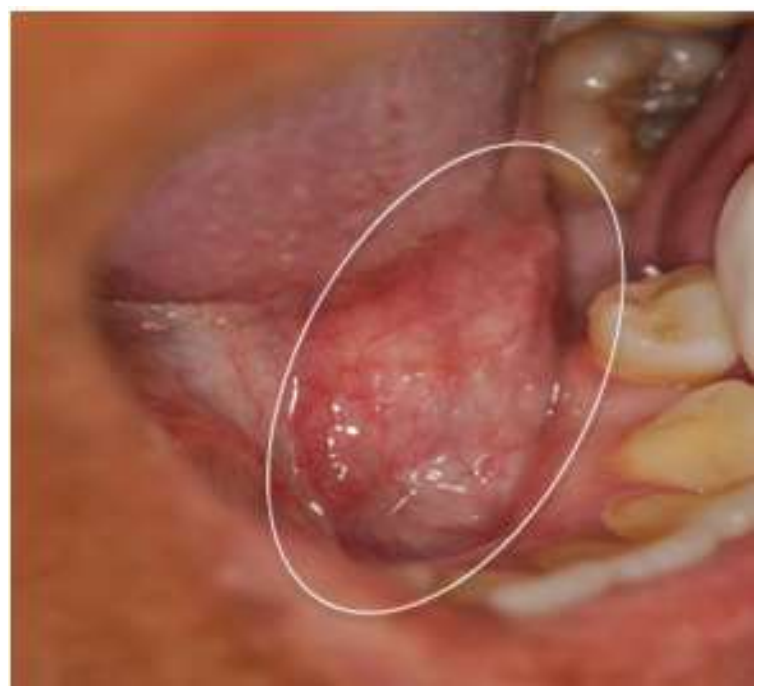

Fonte: Dados da pesquisa.

Figura 2. radiografia panorâmica evidenciando massa radiopaca próximo à região de assoalho bucal.

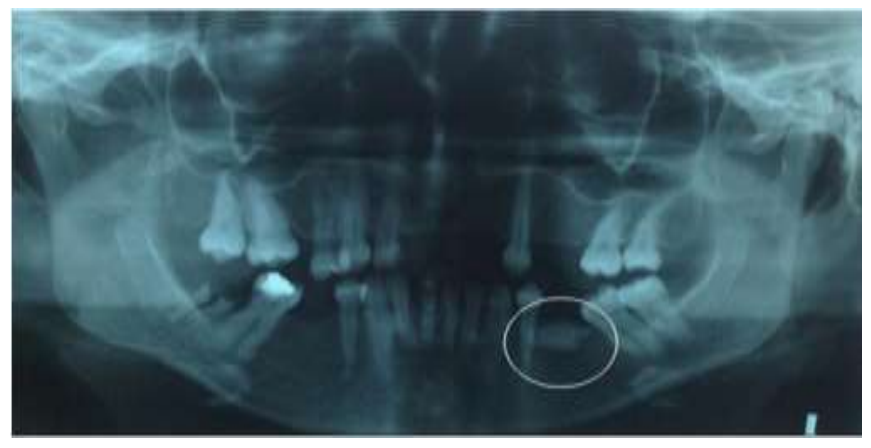

Fonte: Dados da pesquisa.

Figura 3. Radiografia oclusal evidenciando massa radiopaca próximo ao ducto da glândula submandibular.

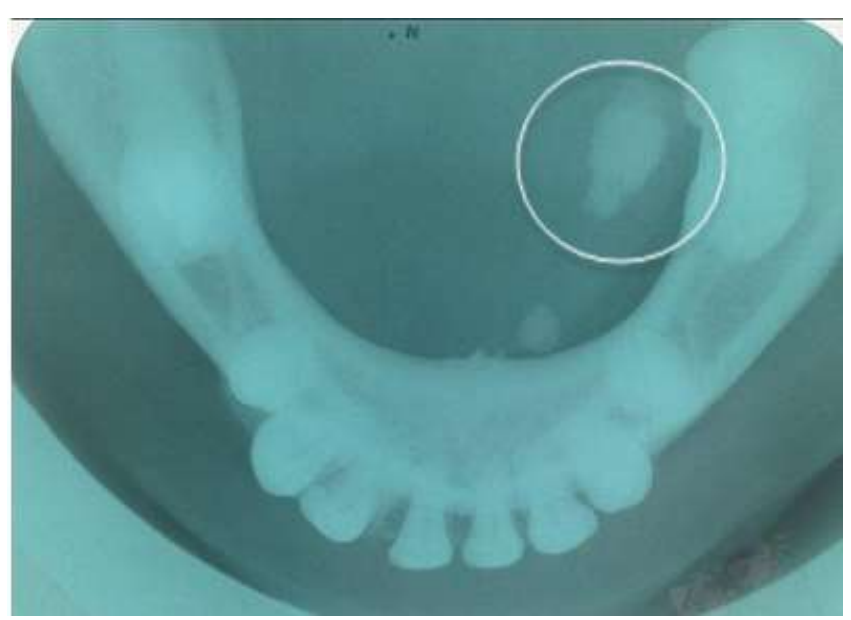

Fonte: Dados da pesquisa. 
Research, Society and Development, v. 10, n. 6, e13410615607, 2021

(CC BY 4.0) | ISSN 2525-3409 | DOI: http://dx.doi.org/10.33448/rsd-v10i6.15607

Figura 4. Telerradiografía lateral.

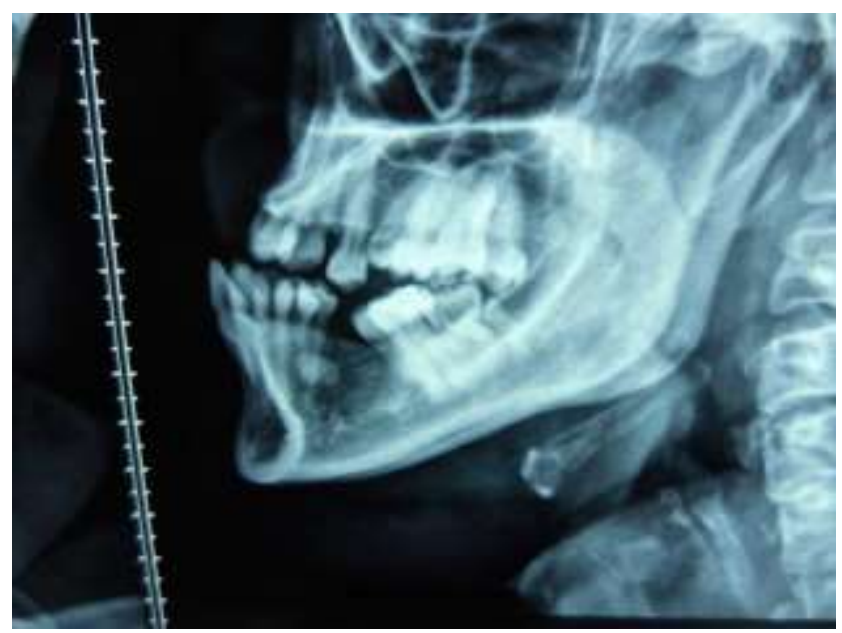

Fonte: Dados da pesquisa.

Figura 5. Ultrassonografia da região de assoalho bucal.

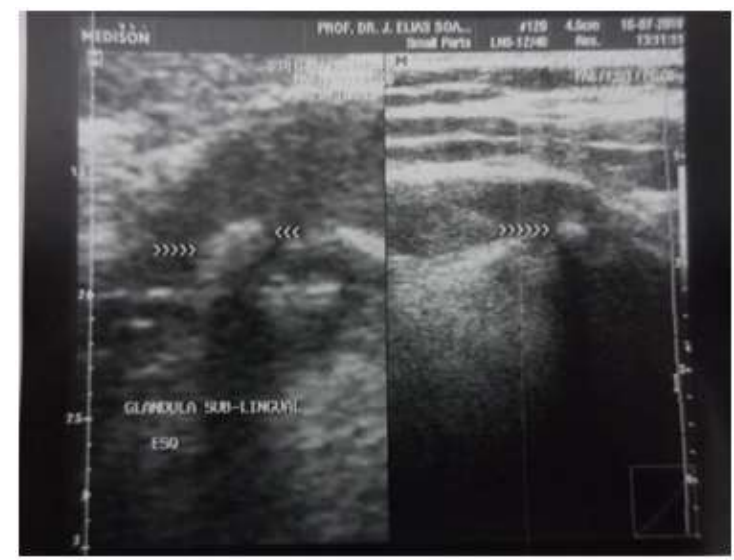

Fonte: Dados da pesquisa.

Figura 6. Lâmina histológica de sialólito (coloração HE tamanho 40x).

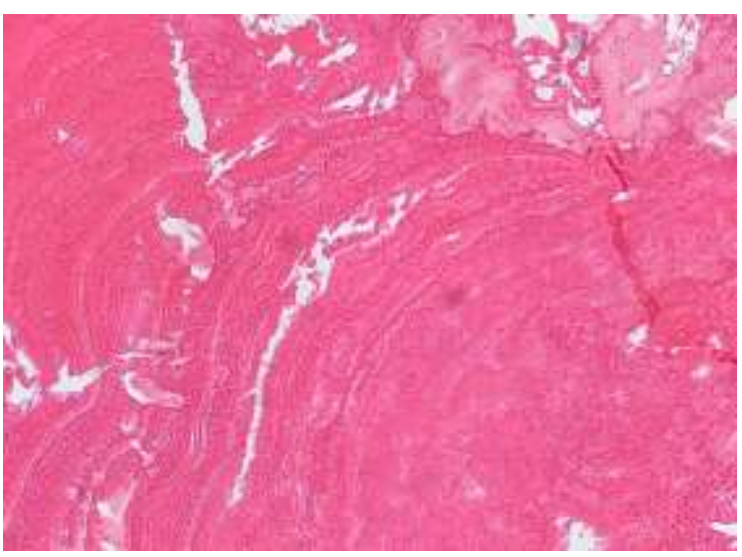


Fonte: Dados da pesquisa.

O resultado do exame anatomopatológico, confirmando o diagnóstico de Sialolitíase foi entregue com 14 dias. A participante da pesquisa foi orientada a retornar ao serviço para consulta de proservação e até o presente momento não compareceu.

\section{Discussão}

Os sialólitos são formados basicamente por componentes de origem orgânica (produtos da saliva como glicoproteínas e mucopolissacarídeos, lipídeos e restos celulares) e origem inorgânica (carbonato-apatita acompanhada de outros sais de cálcio e diferentes tipos de fosfatos, magnésio, ferro, cobre e zinco) (Cobos, Muñoz \& Diaz, 2009). O principal ducto da glândula submandibular pode ser um dos fatores que colabora para o surgimento desta patologia (Kondo et al, 2018), pelo seu curso ser mais tortuoso em relação das glândulas parótidas e sublinguais (Folchini \& Stolz, 2014), bem como pela saliva excretada apresentar uma consistência espessa, contendo fósforo e $\mathrm{Ph}$ alcalino favorável para a formação destes cálculos (Kondo et al, 2018). No presente caso o cálculo estava localizado no interior do ducto da glândula, concordando com a literatura.

Normalmente o sialolito ocorre em indivíduos do sexo masculino (Neville, Damm \& Allen, 2009; Folchini \& Stolz, 2014), com idade média de 50 anos e sem predileção de raça (Neville, Damm \& Allen, 2009). Os sialolitos gigantes possuem ocorrência rara, e quando ocorrem, são mais prevalentes no gênero feminino e se localizam principalmente na região de ductos (Goes, Lima, Carvalho, Queiroz \& Camargo, 2013). Nosso estudo conflita com a literatura, na média de idade, mas corrobora com a ocorrência de cálculos grandes e sua localização, assim como seu tamanho, influenciando no gênero predisponente.

Pacientes com sialolitíase geralmente não sentem dor (Cobos, Muñoz \& Diaz, 2009), mas pode ocorrer geralmente antes, depois ou durante as alimentações por estimular mais o fluxo salivar (Araujo \& Araújo, 1984; Alkurt \& Peker, 2009; Cobos, Muñoz \& Diaz, 2009), apresentando dor que varia de moderada a grave. Esta sintomatología ocorre em 17\% dos casos (Cobos, Muñoz \& Diaz, 2009). Neste relato, a paciente observou volumização da região de assoalho lingual com sintomatologia dolorosa.

Para o direcionamento do diagnóstico do sialolito, os exames complementares de imagens são úteis (Alkurt \& Peker, 2009). O grau de mineralização dos sialolitos são variáveis, e isso faz com que nem todas as lesões sejam vistas em exames radiográficos convencionais. No entanto, eles se apresentam com uma massa radiopaca (Silva, Carneiro, Arantes, Louro \& Rezende, 2020). A melhor forma de se visualizar sialolitos são em radiografias oclusais padrão. Outros exames podem ser utilizados para visualização dos sialolitos, como: sialografia convencional, ultrassonografia, ressonância magnética, cintilografia (Alkurt \& Peker, 2009), sialoendoscopia (Purcell et al, 2017), endoscopia, tomografía computadorizada (TC) e radiografias laterais de crânio para diagnóstico de calcificações em glândula parótida (Cobos, Muñoz \& Diaz, 2009). A sialografia por ressonância magnética obtida bidimensional ou tridimensionalmente é sugerida para se diagnosticar sialolitos, mas não permitem a visualização da parte interna do ducto das glândulas salivares (Alkurt \& Peker, 2009). Já a ultrassonografia representa uma excelente técnica de diagnóstico de primeiro nível porque torna visível os cálculos ductais por evidenciar a nitidez da mineralização tendo uma perfeição de 99\% (Yoshimura, Inoue \& Odagawa, 1989; Oteri, Procopio \& Cicissiù, 2011).

A associação de exames clínicos e radiográficos é o meio de diagnóstico mais utilizado para cálculos em glândulas salivares (Folchini \& Stolz, 2014). Os métodos de diagnósticos escolhidos dependem de características subjetivas da doença, como: tamanho, sinais, localização e sintomas, e das necessidades do paciente. Em geral, as imagens denotam estruturas radiopacas em vários locais (Cobos, Muñoz \& Diaz, 2009)8. No caso clínico apresentado foi observado uma lesão localizada em assoalho bucal de consistência firme e medindo 3,0 × 1,0 x 1,0 cm, sendo acompanhado inicialmente com a radiografia oclusal, seguido por panorâmica, telerradiografia lateral e ultrassonografia.

Diferentes tipos de tratamento podem ser escolhidos de acordo com o tamanho e onde o sialolito está localizado (Alkurt 
\& Peker, 2009). Cálculos menores podem ser "ordenhados" bimanualmente através da saída do ducto (Folchini \& Stolz, 2014; Silva, Carneiro, Arantes, Louro \& Rezende, 2020). Se a calcificação for muito grande pode ser indicada a exérese do cálculo ou ainda da glândula salivar (Alkurt \& Peker, 2009). O acesso é realizado via acesso intraoral através da incisão do assoalho da boca. O ducto é exposto, é realizada a incisão, e o cálculo é removido (Torres et al, 2019). Uma das desvantagens da excisão cirúrgica é que a divulsão dos tecidos pode haver o comprometimento da inervação local (Folchini \& Stolz, 2014). O tratamento escolhido foi a exérese do cálculo a partir de dissecção romba, visto que a ordenha do sialolito foi descartada por conta de suas dimensões. Após a remoção cirúrgica, a espécime foi encaminhada para exame anatomopatológico, que confirmou o diagnóstico de sialolitíase.

\section{Conclusão}

Mesmo sendo uma patologia comum, dentre as que afetam as glândulas salivares, a sialolitíase requer do profissional conhecimento acerca de sua etiologia, formas de diagnosticar e tratar corretamente a doença. O caso relatado mostra que através do exame clínico, imagem e histopatológico, pode-se obter com precisão o diagnóstico da patologia, e pôde-se obter um tratamento conservador da glândula.

Para os próximos trabalhos, sugere-se que, quando possível, se lance mão de tratamentos mais conservadores do que a excisão cirúrgica da lesão, como os métodos de endoscopia e ou litotripsia, a fim de se oferecer uma terapêutica menos invasiva e mais confortável aos pacientes.

\section{Referências}

Abe, A., Kurita, K., Hayashi, H., \& Minagawa M. (2019). A case of minor salivary gland sialolithiasis of the upper lip. Oral MaxillofacSurg, $23,91-94$. https://doi.org/10.1007/s10006-019-00745-6.

Alkurt, M. T., \& Peker, I. (2009). Unusuallylarge submandibular sialoliths: report of two cases. Eur J Dent., 3(2), $135-139$.

Alves, N. S., Soares, G. G., Azevedo, R. S., \& Camisasca, D. R. (2014) Large sialolith in the submandibular gland duct. Rev. Assoc. Paul. Cir. Dent., 68(1), 4053. http://dx.doi.org/10.21270/archi.v8i8.4624.

Araújo, F. A. C., Júnior, O. N. F., Landim, F. S., \& Fernandes, A. (2011). Tratamento cirúrgico de sialólito em glândula submandibular - relato de caso. Rev. Cir. Traumatol. Buco-Maxilo-Fac., 11(4), 13-18.

Araujo, N. S., \& Araújo, V. C. (1984). Patologia Bucal. Artes Médicas.

Aiyekomogbon, I. J., Babatundle, L. B., \& Salam, A. J. (2018). Submandibular sialolithiasis: The roles of radiology in its diagnosis and treatment. Ann Afr Med., 17(4), 221-224. https://doi.org/10.4103/aam.aam_64_17.

Cobos, M. R., Muñoz, Z. C., \& Diaz A .(2009). Sialolitosenconductos y glándulassalivales: Revisión de literatura. Avances en Odontoestomatologia, 25(6), 311-317. https://doi.org/10.4321/S0213-12852009000600002.

Folchini, S., \& Stolz, A. B. (2016). Sialolitos da glândula submandibular: Relato de Caso. Odontol. Clín.-Cient. [online], 15(1), 1-5.

Goes, P. E. M., Lima, V. N., Carvalho, F. S. R., Queiroz, S. B. F., \& Camargo, I. B. (2013). Sialolito gigante em ducto de Wharton: um caso distinto e revisão da literatura. Rev. cir. traumatol. buco-maxilo-fac. 13(4), 81-88.

Jacome, A. M. S. C., \& Abdo, E. N. (2010). Aspectos radiográficos das calcificações em tecidos da região bucomaxilofacial. Odontol. Clín. Cient. [online], 9(1), 25-32.

Kondo N., Yoshihara T., Yamamura, Y., Kusama, K., Sakitani, E., Seo, Y., Tachikawa, M., Kujirai, K., Ono, E., Maeda, Y., Nojima, T., Tamiya, A., Sato, E., \& Nomaka, M. (2018). Treatment outcomes of sialendoscopy for submandibular gland sialolithiasis: The minor axis of the sialolith is a regulative factor for the removal of sialoliths in the hilum of the submandibular gland using sialendoscopy alone. Auris Nasus Larynx, 45(4), 772-776. https://doi.org/10.1016/j.anl.2017.09.003.

McGurk, M., Makdissi,. J., \& Brown, J. E. (2004). Intra-oral removal of stones from the hilum of the submandibular gland: report of technique and morbidity. Int J Oral MaxillofacSurg, 33, 683-6. https://doi.org/10.1016/j.ijom.2004.01.024.

Neville, B. W., Damm, D. D. Allen, C. M., \& Bouquot, J. E. (2009). Patologia Oral e Maxilofacial (3a ed). Guanabara-Koogan.

Oteri, G., Procopio, R. M., \& Cicissiù, M. (2011). Giant Salivary Gland Calculi (GSGC): Report Of Two Cases Op Dentistry. The Open Dentistry Journal, 5(1), 90-95. https://10.2174/1874210601105010090. 
Research, Society and Development, v. 10, n. 6, e13410615607, 2021

(CC BY 4.0) | ISSN 2525-3409 | DOI: http://dx.doi.org/10.33448/rsd-v10i6.15607

Pereira A. S. et al. (2018). Metodologia da pesquisa científica. UFSM.

Purcell, Y. M., Kavanagh, R. G., Cahalane, A. M., Carroll, A. G., Khoo, S. G., \& Killeen, R. P. (2017). The Diagnostic Accuracy of Contrast-Enhanced CT of the Neck for the Investigation of Sialolithiasis. American Journal of Neuroradiology, 38(11), 2161-2166. https://doi.org/10.3174/ajnr.A5353.

Silva, F. B. M., Carneiro, N. S., Arantes, E. R., Louro, R. S. \& Resende, R. F. B. (2020). Tratamento cirúrgico de sualolito de grandes proporções em glândula submandibular: relato de caso. Revista Fluminense de Odontologia, (53), 18-28. https://periodicos.uff.br/ijosd/article/view/39862/22945.

Torres, L. H. S., Santos, M. S., Diniz, J. A., Uchôa, C. P., Silva, J. A. A., Pereira Filho, V. A., \& Oliveira e Silva, E. D. (2019) Remoção Cirúrgica de sialolito em glândula submandibular: relato de caso. Arch Health Invest, 8(8), 421-424. https://www.archhealthinvestigation.com.br/ArcHI/article/view/4624/pdf

Yoshimura, Y., Inoue, Y., \& Odagawa, T. (1989). Sonographic examination of sialolithiasis. J Oral MaxillofacSurg, 47, 907-912. https://10.1016/02782391(89)90372-8. 